\title{
Identification of the Thermoelectric Cooler Using Hybrid Multi-Verse Optimizer and Sine Cosine Algorithm Based Continuous-Time Hammerstein Model
}

\author{
Julakha Jahan Jui ${ }^{1}$, Mohd Ashraf Ahmad ${ }^{1}$, Mohamed Sultan Mohamed \\ Ali $^{2}$, Mohd Anwar Zawawi ${ }^{1}$, Mohd Falfazli Mat Jusof ${ }^{1}$ \\ ${ }^{1}$ Faculty of Electrical and Electronics Engineering Technology (FTKEE), Universiti Malaysia Pahang \\ (UMP), Pekan, Pahang, 26600, Malaysia \\ ${ }^{2}$ School of Electrical Engineering, University Teknologi Malaysia (UTM), Johor Bahru, Johor, 81310, \\ Malaysia \\ E-mails:_julakha.ump@gmail.com_mashraf@ump.edu.my_sultan_ali@fke.utm.my \\ mohdanwar@ump.edu.my_mfalfazli@ump.edu.my
}

\begin{abstract}
This paper presents the identification of the ThermoElectric Cooler (TEC) plant using a hybrid method of Multi-Verse Optimizer with Sine Cosine Algorithm ( hMVOSCA) based on continuous-time Hammerstein model. These modifications are mainly for escaping from local minima and for making the balance between exploration and exploitation. In the Hammerstein model identification a continuoustime linear system is used and the hMVOSCA based method is used to tune the coefficients of both the Hammerstein model subsystems (linear and nonlinear) such that the error between the estimated output and the actual output is reduced. The efficiency of the proposed method is evaluated based on the convergence curve, parameter estimation error, bode plot, function plot, and Wilcoxon's rank test. The experimental findings show that the hMVOSCA can produce a Hammerstein system that generates an estimated output like the actual TEC output. Moreover, the identified outputs also show that the hMVOSCA outperforms other popular metaheuristic algorithms.
\end{abstract}

Keywords: Thermoelectric cooler, Hammerstein system, Metaheuristics Algorithm, multi-verse optimizer, Sine Cosine Algorithm, optimization.

\section{Introduction}

A significant problem in the field of a control system is temperature regulation. Nowadays, power and temperature control becomes one of the challenging tasks in this field [1-3]. In the fields of medical, military, aerospace, and precision instruments, the temperature regulation strategy is broadly implemented [4]. Mainly, the heating as well as the cooling plants and processes are generally discrete in conventional temperature control engineering. The heating process transforms 
electrical power or chemical power into thermal energy, which is commonly used in industry for electrical heating elements [5]. Based on the applications of the heating process, the refrigeration systems are typically made up of air-cooled, water-cooled, and compressed refrigeration [6,7]. The main disadvantage of the mentioned temperature regulation approaches is that the heating and cooling mechanism cannot be transformed effectively. Compared to conventional temperature regulation techniques, ThermoElectric Cooler (TEC) temperature controller has the benefits of low volume, lightweight, long lifetime, no physical motions, no noise, no refrigerant, fast refrigeration, highly accurate temperature regulation, and no environmental contamination [8]. For the cooling of electronic instruments such as CPUs, infrared sensors, thermocouple thermometer, and refrigerators, where the thermoelectric coolers have been frequently used $[9,10]$. Usually, the temperature at the thermoelectric module's cold side is maintained by using constant and stabilized value under varying hot-side and atmospheric temperatures where the electronic component such as the infrared sensor is installed. This is based on a good technique of temperature control. However, a major error in temperature regulation is normally occurred due to the inaccuracy of modelling. Therefore, a proper identification method is necessary to obtain a precise model of such a complex plant or process.

On the other hand, the block-oriented models for the identification of nonlinear system have become a prominent strategy to model such type of complex plants [11]. There are mainly three types of block-oriented nonlinear models present, which are the Hammerstein model, Wiener model, and Hammerstein-Wiener model. Among all these nonlinear system identification models, the Hammerstein model is the popular one because of its easy model structure and common usage to identify nonlinear systems [12]. Hammerstein model has also been applied to model many actual plants and processes including Solid Oxide fuel cell [13], turntable servo system [14], amplified piezoelectric actuators [15] multi-axis piezoelectric micro-positioning stages [16], and pneumatic muscle actuators [17]. Moreover, there are many traditional identification methods that have been used for the identification of the Hammerstein models such as the iterative method [18], the subspace method [19], the least square method [20] and the blind approach [21]. When these methods are used to identify the Hammerstein model, the process of parameter estimation is converted into a multidimensional problem in which the parameters of the linear and nonlinear system are considered as a decision variable. In these situations, the complexity of the traditional method appears to generate multimodal error surfaces which present significant challenges in minimizing their cost functions. This limitation can be overcome by using various optimization algorithms. Furthermore, many researchers also utilize the optimization algorithms to identify the Hammerstein models, such as the Cuckoo search Algorithm [22], Particle Swarm optimization [23], Gravitational Search Algorithm [24], and Sine Cosine Algorithm [25]. From the above literature, various weaknesses are inevitable in the previous studies, such as:

1. Most of the Hammerstein models used in their research are based on the discrete-time model, while the continuous-time model can easily represent several real plants. 
2. Their techniques suffer from a problem of over-parameterization, where the parameters of the nonlinear and linear components are redundant and can have high computational loads.

However, a more common group of Hammerstein model can be solved by our research model by considering a continuous-time transfer function in the linear subsystem. In addition, it is important to avoid the redundancy of the nonlinear and linear subsystem parameters in order to reduce the computational workload. On the other hand, Multi-Verse Optimizer (MVO) [26] has now become a popular optimization technique that has addressed different types of technical problems [27] such as reactive power dispatch problem, parameter extraction of a photovoltaic generating unit, identify the optimal parameter of proton exchange membrane fuel cell and finding the solution of load frequency control problem [28-32]. Nevertheless, compared to MVO, the recent optimization techniques are very complicated, which can lead to a high initial coefficient setting and increases the computational load. Therefore, this motivates us to see the efficacy of the MVO from the actual experimental data in modelling the thermoelectric cooler plant. In addition, based on our preliminary work on this issue, the standard MVO is still unable to provide the thermoelectric cooler model with high accuracy, as it can fall into local minima and imbalance between exploration and exploitation. In this research, we intend to develop a new hybrid algorithm to solve the problems of the conventional MVO by combining the MVO and Sine Cosine Algorithm (SCA). The SCA relies mainly on the functions of the sine and cosine to find the solutions to the given problem [33]. The SCA has been extended to many applications on the basis of these behaviours, including feature selection [34], oil consumption forecasting [35] and image segmentation [36]. Then, this encourages us to hybridize the standard MVO algorithm with the SCA algorithm so that the local minimum problem and the imbalance between the exploration and exploitation can be solved to obtain a better accuracy of the thermoelectric cooler model.

In this paper we propose a hybrid algorithm called hybrid Multi-Verse Optimizer and Sine Cosine Algorithm (hMVOSCA) for the identification of the thermoelectric cooler plant using the Hammerstein model where the conventional MVO is hybrid with SCA. This hybridization is useful to achieve a better balance between exploration and exploitation and has the advantage of escaping from local minima and improving optimization accuracy. The hMVOSCA based method is used to tune the coefficients of both Hammerstein models subsystem so that the error between the identified and the actual output is reduced. The evaluation of the experimental finding is investigated based on the convergence curve of the objective function, parameter estimation error, bode plot of the linear subsystem, the function plot of the nonlinear subsystem, and the Wilcoxon's rank test. The results are also compared with the most popular optimization algorithms such as the Particle Swarm Optimizer (PSO), Grey Wolf Optimizer (GWO), Ant Lion Optimizer (ALO), MVO, and SCA.

The rest of the paper is organized as follows. In Section 2, the conventional MVO and the proposed identification scheme called hybrid MVOSCA are described. Section 3 discusses the problem formulation. Section 4 shows the experimental 
results and the analysis of the efficiency of the proposed method. Finally, some concluding remarks of the investigation are discussed in Section 5.

\section{Hybrid Multi-Verse Optimizer and Sine Cosine Algorithm}

The proposed algorithm hMVOSCA is explained in this section. Firstly, a brief overview of the MVO is given, and secondly, the procedures for modifying the conventional MVO Algorithm are described.

\subsection{Overview of Multi-Verse Optimizer Algorithm}

The Multi-Verse Optimization (MVO) technique is one of the new swarm intelligence technique proposed by Mirjalili et al. [26]. MVO Algorithm is mainly influenced by the Big Bang theory and Quantum physics. The multi-verse theory describes how various universes are formed by the big bangs and how the generated universes communicate with each other via various types of holes, such as white hole, black hole, and wormhole. There is an inflation rate for each universe that induces its expansion across space. According to the MVO Algorithm, the universe and the solution of each element define an object in that universe, and to represent the solution efficiency, each solution has an inflation rate which is determined by the subsequent objective function. When white holes appear, a better objective value is given to a solution, while a poor objective value is given to the solution if the black holes appear. The attribute values of the good solutions are changed to poor solutions when more correlations between white and black holes happen. Fig. 1 shows the diagram of the MVO Algorithm and the detailed of the MVO Algorithm is explained by firstly considering an optimization problem as

$$
\min _{z_{i}(i=1,2, \ldots, n)} I\left(z_{i}\right),
$$

where $I: R^{n} \rightarrow R$ is the inflation rate which is the objective function, and $z_{i} \in R^{n}$ is the design variable of the universe $i$.

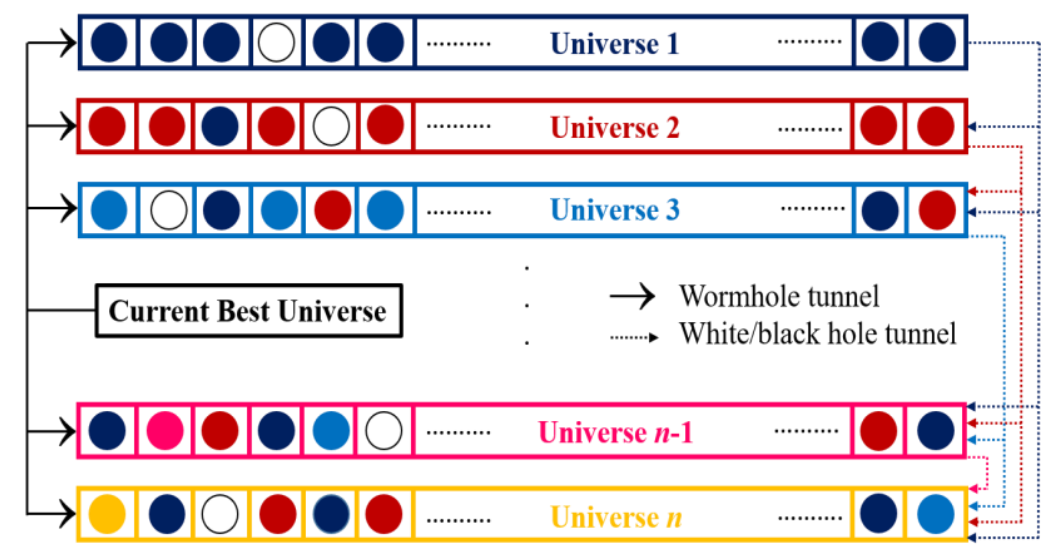

Fig. 1. Procedure of the MVO Algorithm 
The MVO Algorithm's main mathematical formula relies on (2) and (3), as defined below:

$$
z_{i j}= \begin{cases}z_{k j} & r_{1}<\operatorname{NRI}\left(M_{i}\right), \\ z_{i j} & r_{1} \geq \operatorname{NRI}\left(M_{i}\right),\end{cases}
$$

where $z_{k j}$ is the $j$-th element of $k$-th universe, $z_{i j}$ refers the $j$-th element of $i$-th universe selected by a roulette wheel selection mechanism, and $r_{1}$ is a randomly generated number, $r_{1} \in(0,1)$; $\operatorname{NRI}\left(M_{i}\right)$ defines the $i$-th universes normalized inflation rate, $M_{i}$ shows the $i$-th universe. The inflation rates are standardized after the universe evaluation, to get their values between 0 and 1 .

Since the wormholes are being used to transfer elements randomly between the universes and because of their inflation rate, they are generated between a universe as well as the best universe. The following equation shows the mechanism of that procedure:

$$
z_{i j}=\left\{\begin{array}{cl}
Z_{j}+\operatorname{TDR}\left(\left(\mathrm{ub}_{j}-\mathrm{lb}_{j}\right) r_{2}+\mathrm{lb}_{j}\right) & \text { if } r_{3}<0.5 \text { and } r_{4}<\mathrm{WEP}, \\
Z_{j}-\operatorname{TDR}\left(\left(\mathrm{ub}_{j}-\mathrm{lb}_{j}\right) r_{2}+\mathrm{lb}_{j}\right) & \text { if } r_{3} \geq 0.5 \text { and } r_{4}<\mathrm{WEP}, \\
z_{i j} & \text { if } r_{4} \geq \mathrm{WEP},
\end{array}\right.
$$

where $Z_{j}$ defines the $j$-th element of the best universe obtained so far, $z_{i j}$ defines the $j$-th element of $i$-th universe, Wormhole Existence Probability (WEP) and Traveling Distance Rate (TDR) are the coefficients defined by the following equations (4) and (5), $r_{2}, r_{3}, r_{4}$ are the independently generated numbers, and their values are between 0 and $1 ; \mathrm{ub}_{j}$ and $\mathrm{lb}_{j}$ are the upper bound and lower bound limits of the elements. Equation (3) has been structured specifically to update the position of the solutions,

$$
\begin{gathered}
\mathrm{WEP}=l+t_{\text {iter }}\left(\frac{h-l}{T_{\text {iter }}}\right), \\
\mathrm{TDR}=1-\frac{t_{\text {iter }}^{\frac{1}{e}}}{T_{\text {iter }} r^{\frac{1}{e}}}
\end{gathered}
$$

where minimum value is defined as $l$, and $h$ define the maximum value. Also, the current iteration is defined as $t_{\text {iter }}$ and maximum iteration is defined as $T_{\text {iter }}, e$ is defined as the exploitation factor over the iterations and the higher value of $e$ indicates more accurate exploitation. WEP defines the probability of existence of the wormhole in a universe, and the TDR defines the distance rate of the transferred elements through the wormhole tunnel around the best universe. In order to emphasize the exploitation process, the WEP parameter increases linearly during the iterations and the TDR parameter reduces the traveling distance rate so that the elements can be carried around the best universe, which upturns the local search over iterations. The detailed description of the convolutional MVO algorithm is given in [26]. 


\subsection{Proposed algorithm}

Although the MVO algorithm has several benefits, it also has a few drawbacks, such as fall into local minima region and low exploration and exploitation [37]. To handle those limitations, a new position updating mechanism is proposed where the sine and the cosine function of the Sine Cosine Algorithm (SCA) [33] is adopted. Further details are discussed in this section of the newly proposed MVO version called hybrid Multi-Verse Optimizer and Sine Cosine Algorithm (hMVOSCA) based on MVO and SCA by taking advantage of the algorithm SCA.

In this paper, the proposed hMVOSCA based method performs the searching of the new position by the sine and cosine function of the algorithm SCA more effectively. When universes seem unable to find the best solutions, they re-form the solution so that the best universe search process is impacted, and this will help to avoid the local minima with the Sine and Cosine function of the algorithm SCA. In general, the work in this area of study is designed to enhance the efficiency of the conventional MVO method by improving its next position selection mechanism. In this paper, we basically perform several modifications upon the conventional MVO. Our first modification is for solving the local minima problem. Here, it is considered that the current universe would be constructed by taking the average of the previous universe and the best universe obtained so far to ensure local improvements across the universes and the probability of improving the inflation rate and helping the algorithm hMVOSCA to get the better inflation rate of the universe and get back from the local minima zone. The second modification is for balancing the exploitation and exploration. Here, the next position of the solution is determined by using the sine and cosine function of the algorithm SCA. The cyclic structure of the role of the sine and cosine makes it possible to re-position a solution around another solution. This will ensure the exploitation of the defined space between the two solutions. The solutions should also be able to search outside the space between their corresponding destinations to explore the search space.

The mathematical equation of selecting the next position of the solution is as follows:

$$
z_{i j}=\left\{\begin{array}{cl}
B_{i j}+\operatorname{TDR}\left(\sin \left(2 \pi r_{5}\right)\left|2 r_{6} Z_{j}-z_{i j}\right|\right) & \text { if } r_{3}<0.5 \text { and } r_{4}<\mathrm{WEP}, \\
B_{i j}+\operatorname{TDR}\left(\cos \left(2 \pi r_{5}\right)\left|2 r_{6} Z_{j}-z_{i j}\right|\right) & \text { if } r_{3} \geq 0.5 \text { and } r_{4}<\mathrm{WEP}, \\
z_{i j} & \text { if } r_{4} \geq \mathrm{WEP}, \\
B_{i j}=\frac{z_{j}+z_{i j}}{2}, &
\end{array}\right.
$$

where $B_{i j}$ is the average universe position, generated by selecting the average position between the current universe position and the position of the best universe obtained so far. The coefficients $r_{5}, r_{6}$ are the randomly generated numbers between 0 and 1 . The parameter $r_{5}$ determines distance of the movement should be towards the destination or outwards the destination. In order to stochastically emphasize $\left(r_{6}>1\right)$ or enhance $\left(r_{6}<1\right)$ the effect of the target point in defining the range, the parameter $r_{3}$ introduces a random weight for the location. Finally, in switching 
between sine and cosine functions in (6), the parameter $r_{3}$ is used. The flowchart of the complete procedure of the algorithm hMVOSCA is shown in Fig. 2.

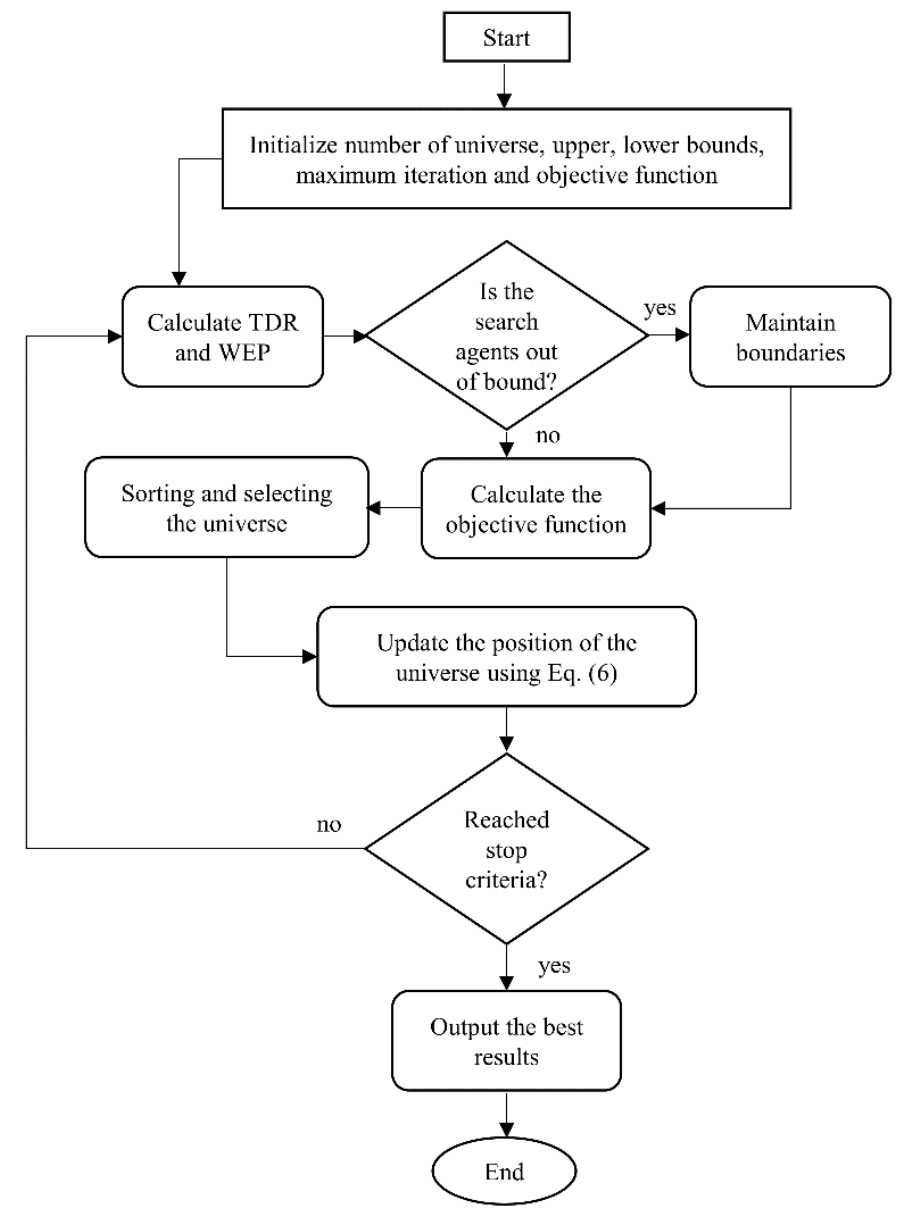

Fig. 2. Complete process of the algorithm hMVOSCA

\section{Problem formulation}

In this section, the proposed hMVOSCA for the identification of ThermoElectric Cooler (TEC) plant based on continuous-time Hammerstein model is discussed. Initially, to identify the thermoelectric cooler a problem formulation is explained and after that, based on the Hammerstein model, the application of the hMVOSCA method to identify the TEC plant is shown.

The block-diagram of TEC model identification using the Hammerstein model is shown in Fig. 3. The model is composed of two subsystems such as the nonlinear static function defined as $f$ and a dynamic linear system defined as $D$ and its differential operator $\left(p: \frac{d}{d t}\right)$ can be defined by: 


$$
D(p)=\frac{B(p)}{A(p)}=\frac{b_{m} p^{m}+b_{m-1} p^{m-1}+\cdots+b_{0}}{p^{n}+a_{n-1} p^{n-1}+\cdots+a_{0}}
$$

the nonlinear static function $f$ is defined as

$$
f(x(t))=\sum_{j=1}^{q} c_{j} \delta_{j}\left[c_{j+1} x(t)\right]
$$

where $\delta[$.$] is known as the nonlinear function, c_{j}$ is the coefficient of the nonlinear function, the input signal of the model is defined as $x(t)$, and the output signal $y(t)$ is described as $\tilde{y}(t)$ when the noise signal $\varepsilon(t)$ is integrated with the output signal. Finally, we may define $\tilde{y}(t)$ as

$$
\tilde{y}(t)=D(p) f(x(t))+\varepsilon(t) .
$$

For the identification problem we need to assume few terms such as:

Assumption 1. The symbols $m, n$ and $q$ are known.

Assumption 2. Coefficients $a_{j}, j=0,1, \ldots, n-1, b_{j}, j=0,1, \ldots, m$, and $c_{j}$, $j=1,2, \ldots, q$, are considered to be positive real numbers.

Assumption 3. $f(0)=0$.

Assumption 4. The linear transfer function $D(p)$ is asymptotically stable.

Assumption 5. The first design variable $b_{m}=1$, such that $D(p)$ and $f(x(t))$ can be achieved uniquely.

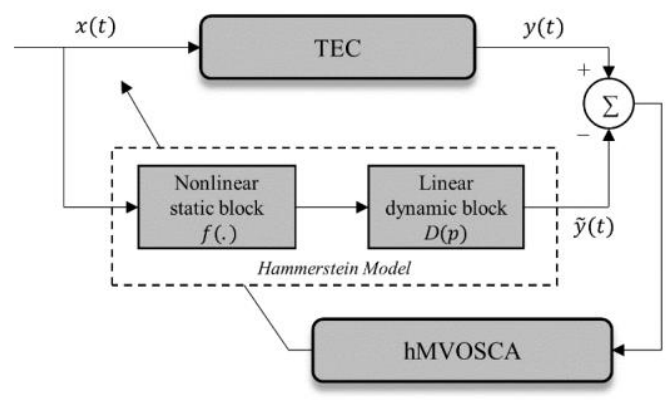

Fig. 3. TEC identification block-diagram using continuous-time Hammerstein system

To evaluate the estimated model, the following objective function is implemented,

$$
J(\dot{D}, \hat{f})=\sum_{k=0}^{N}\left(\tilde{y}\left(k t_{\mathrm{s}}\right)-\dot{y}\left(k t_{\mathrm{s}}\right)\right)^{2},
$$

where $t_{s}$ is the sampling rate for $(x(t), \tilde{y}(t)), t=0, t_{\mathrm{s}}, 2 t_{\mathrm{s}}, \ldots, N t_{\mathrm{s}}, N=0,1,2 \ldots$ The estimated symbols of linear and nonlinear subsystems $D$ and $f$ are defined as $D$ and $f$, respectively, and the estimated output is defined as $\dot{y}=\dot{D}(p) f(x(t))$.

Finally, the identification problem is explained as follows.

Problem 1. For the continuous-time Hammerstein system in Fig. 3, the inputoutput data $(x(t), \tilde{y}(t)), t=0, t_{\mathrm{s}}, 2 t_{\mathrm{s}}, \ldots, N t_{\mathrm{s}}$, are given. Then, find $\dot{D}$ and $\hat{f}$ such that $J(\dot{D}, f)$ is minimized.

Furthermore, the algorithm hMVOSCA for the identification of Hammerstein system is applied to solve Problem 1. Using hMVOSCA, the identification problem in (11) is updated to the objective function as

$$
g(\boldsymbol{\theta})=\sum_{k=0}^{N}\left(\tilde{y}\left(k t_{\mathrm{s}}\right)-\dot{y}\left(\left(k t_{\mathrm{s}}\right)\right)^{2},\right.
$$


for the design variable

(13) $\boldsymbol{\theta}=\left[b_{0}, b_{1}, \ldots, b_{m}, \dot{a}_{0}, \dot{a}_{1}, \ldots, \dot{a}_{n-1}, \dot{c}_{1}, \dot{c}_{2}, \ldots, \dot{c}_{q}\right]$.

It is required to determine the value of $g(\boldsymbol{\theta})$ for a specified $\boldsymbol{\theta}$, which is generalized to measure $\left(\tilde{y}\left(k t_{\mathrm{s}}\right)\right.$, that is shown as follows. At first the input signal $x(t)$ is produced with a zero-order hold and $x(t), t=0, t_{\mathrm{s}}, 2 t_{\mathrm{s}}, \ldots, N t_{\mathrm{s}}$. Then, we estimate

$$
\dot{y}(t)=\frac{\dot{b}_{m} p^{m}+\dot{b}_{m-1} p^{m-1}+\cdots+\dot{b}_{0}}{p^{n}+\dot{a}_{n-1} p^{n-1}+\cdots+\dot{a}_{0}} f(x(t)),
$$

in the continuous-time environment. After that $y^{\prime}(t)$ is sampled to $y\left(k t_{\mathrm{s}}\right)$ at a constant sampling rate $t_{\mathrm{s}}$ for $k=0,1, \ldots, N$. Finally, using hMVOSCA based technique, a solution to Problem 1 is obtained. The complete process may be summarized as follows:

Step 1. Identify the design variable $\boldsymbol{\theta}$.

Step 2. Apply hMVOSCA based method by setting $:=z_{i}$ and $J(D, f)=I_{i}$.

Step 3. Once the maximum iteration $T_{\text {iter }}$ is reached, we achieve $z^{*}=Z$.

Then, $\boldsymbol{\theta}^{*}=z^{*}$ is the solution for Problem 1 .

\section{Experimental results and discussion}

The efficacy of the hMVOSCA based approach for TEC system identification using the Hammerstein system is illustrated in this section. The performance criteria for this identification are as follows:

1. Convergence curve of the objective function from 25 trials, evaluation of parameter estimation error. The mathematical formula of parameter estimation error $\zeta$ is written as

$$
\zeta=\left\|\frac{\theta_{1}-\bar{\theta}_{1}}{\bar{\theta}_{1}}, \ldots, \frac{\theta_{n}-\bar{\theta}_{n}}{\bar{\theta}_{n}}\right\|_{2}
$$

where $\bar{\theta}_{1}$ is the $i$-th element of the design variable vector $\bar{\theta}$ and $\theta_{i}$ is the $i$-th element of the identified design variable $\theta$.

2. Bode plot of the estimated linear system $D(p)$ and the function plot of the estimated nonlinear function $f(x(t))$.

3. The investigation of statistical performance value of the objective function and parameter estimation error values from 25 independent trials based on the mean, best, worst, and standard deviation (Std.), between hMVOSCA, PSO, GWO, ALO, MVO and SCA for different noise levels.

The TEC system has a multi-mode frequency in its linear dynamic system. Note that the nonlinear subsystem is dedicated to identifying the heat condition at the coldend plate. Both the linear and the nonlinear subsystems are given by

$$
\begin{gathered}
D(p)=\frac{p+0.1323}{p^{2}+0.5964 p+0.00855} \\
f(x(t))=-0.4141 \sinh (0.5 x(t)) .
\end{gathered}
$$

Note that the transfer function in (16) is taken from the validated TEC model in [38]. In this experiment, a varying amplitude of PRBS signal is used for $x(t)$, and the amplitude of the signal is varied in the range of $[-1,1]$. Here, we set two levels of white noise with variances $\sigma_{v}^{2}=0.001$ and $\sigma_{v}^{2}=0.0025$. The signal $y(t)$ is sampled at $t_{\mathrm{s}}=1 \times 10^{-3}$ over $N=24,000$. The coefficients of hMVOSCA that are 
used to identify the TEC are $\mathrm{lb}_{j}=-0.5$ for all $j, \mathrm{ub}_{j}=1$ for all $j, T_{\text {iter }}=400$, $n=25, h=1, l=0.2, p=4$, and $d=5$. The coefficient setting of the other metaheuristics algorithms are shown in Table 1.

Table 1. Coefficients setting of PSO, GWO, ALO, MVO and SCA based method

\begin{tabular}{|c|c|c|c|c|c|}
\hline Algorithms & PSO & GWO & ALO & MVO & SCA \\
\hline \multirow{4}{*}{ Coefficients } & $W_{\max }=0.9$ & & & $h=1$ & \\
& $W_{\min }=0.4$ & $a=2$ & --- & $l=0.2$ & $a=2$ \\
& $c_{1}=1.45$ & & & $p=6$ & \\
& $c_{2}=1.45$ & & & \\
\hline
\end{tabular}

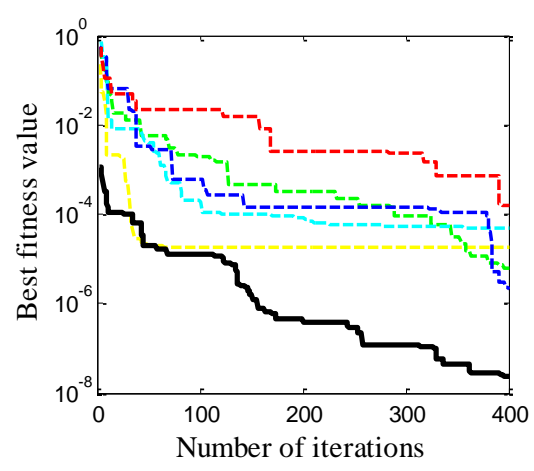

(a) No noise

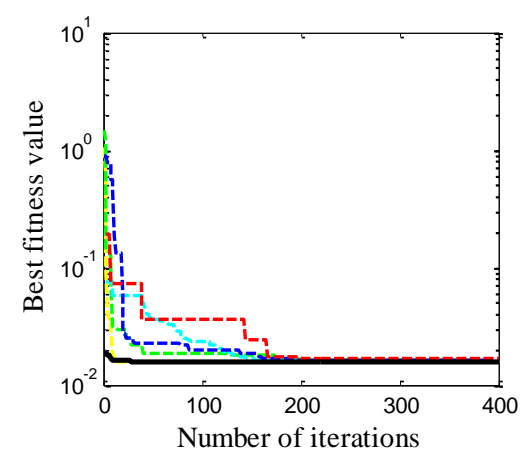

(b) $\sigma_{v}^{2}=0.001$

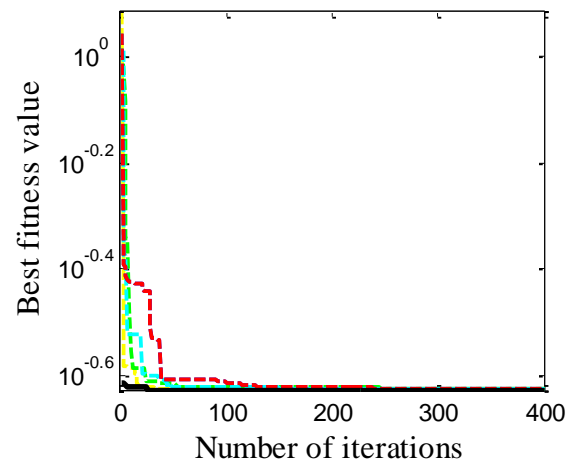

(c) $\sigma_{v}^{2}=0.0025$

$$
\text { PSO ------ GWO ------ ALO ------ MVO ------ SCA } \longrightarrow \text { hMVOSCA }
$$

Fig. 4. Convergence curve of best objective function from 25 trials with several noise variances

The convergence curve of best objective function with no noise variance and two noise variances $\sigma_{v}^{2}=0.001$ and $\sigma_{v}^{2}=0.0025$ for the algorithms hMVOSCA, PSO, GWO, ALO, MVO and SCA based method are shown in Figs 4a, b and c, respectively. These curves illustrate that the hMVOSCA based method can successfully minimize the objective function to obtain the best identified design variable values as compared to the other methods. The best identified design variables 
and its corresponding parameter estimation error with several noise variances for hMVOSCA are tabulated in Table 2.

Table 2. Best identified design variables and its corresponding parameter estimation error with noise variances using hMVOSCA based method

\begin{tabular}{|c|c|c|c|c|c|}
\hline \multirow{2}{*}{$\boldsymbol{\theta}$} & \multirow{2}{*}{$\begin{array}{c}\text { Design } \\
\text { variable }\end{array}$} & \multicolumn{3}{|c|}{$\boldsymbol{\theta}$} & \multirow{2}{*}{$\overline{\boldsymbol{\theta}}$} \\
\hline & & No Noise & $\sigma_{v}^{2}=0.001$ & $\sigma_{v}^{2}=0.0025$ & \\
\hline$\theta_{1}^{*}$ & $\hat{b}_{0}$ & 0.1378 & 0.2095 & 0.1713 & 0.1323 \\
\hline $\boldsymbol{\theta}_{2}^{*}$ & $\dot{a}_{1}$ & 0.6069 & 0.6897 & 0.5726 & 0.5964 \\
\hline$\theta_{3}^{*}$ & $\dot{a}_{0}$ & 0.0096 & 0.0233 & 0.0195 & 0.00855 \\
\hline $\boldsymbol{\theta}_{4}^{*}$ & $\dot{c}_{1}$ & -0.4346 & -0.3074 & -0.4724 & -0.4141 \\
\hline$\theta_{5}^{*}$ & $c_{2}$ & 0.4784 & 0.6467 & 0.4024 & 0.5 \\
\hline & $\xi$ & 0.1466 & 1.8738 & 1.3374 & - \\
\hline
\end{tabular}

Furthermore, Figs 5 and 6 clearly demonstrate the nonlinear function $f(x(t))$ plot and the bode plot of the linear dynamic system $D(p)$ with various noise variances. It indicates that the hMVOSCA based method can closely identify the true plot response of the nonlinear function $f(x(t))$ especially when there are no noise variances. However, there is a slightly small variation between $f(x(t))$ and $f(x(t))$ responses for $\sigma_{v}^{2}=0.001$ and $\sigma_{v}^{2}=0.0025$. Similarly, the hMVOSCA based method can also provide an almost accurate $D(p)$ when there are no noise variances. This can be clearly seen from the responses of the bode plot in Fig. 6. However, there is a small deviation in the bode plot response of noise levels $\sigma_{v}^{2}=0.001$ and $\sigma_{v}^{2}=0.0025$ particularly at the low frequency region.

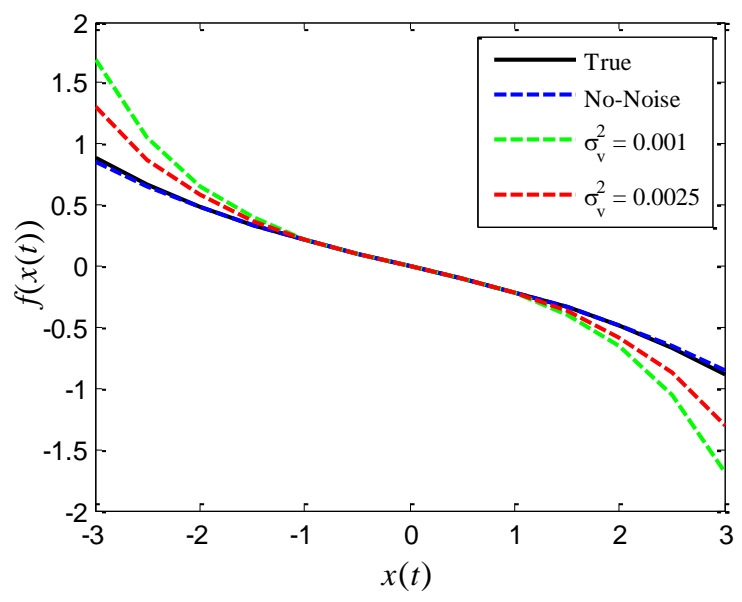

Fig. 5. Identified nonlinear function $\boldsymbol{f}(\boldsymbol{x}(\boldsymbol{t}))$ with no noise and two noise variances 


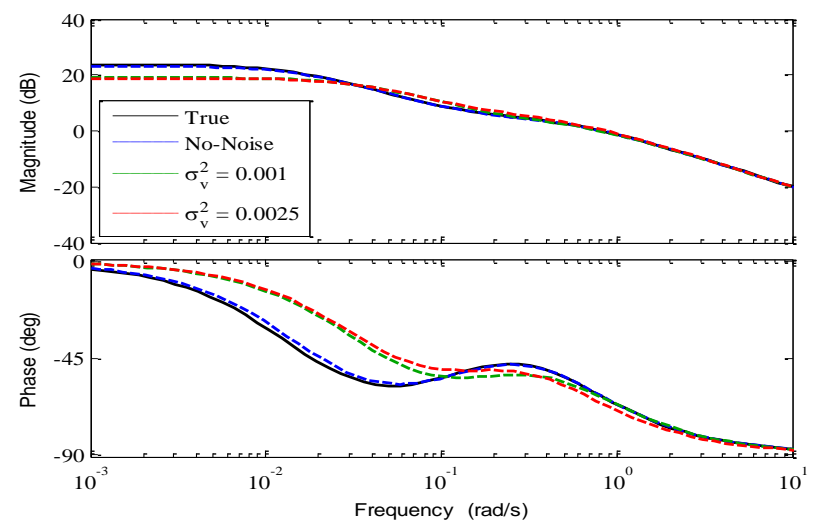

Fig. 6. Identified linear dynamic system $\boldsymbol{D}(\boldsymbol{p})$ with no noise and two noise variances

Table 3. Best identified design variables and its corresponding parameter estimation error with noise variances

\begin{tabular}{|c|c|c|c|c|c|c|c|c|c|c|}
\hline \multirow{2}{*}{\multicolumn{2}{|c|}{$\frac{\text { Algorithm }}{\sigma_{v}^{2}}$}} & \multicolumn{3}{|c|}{ hMVOSCA } & \multicolumn{3}{|c|}{ PSO } & \multicolumn{3}{|c|}{ GWO } \\
\hline & & $\mathrm{NN}$ & 0.001 & 0.0025 & NN & 0.001 & 0.0025 & NN & 0.001 & 0.0025 \\
\hline \multirow{4}{*}{$J(\theta)$} & Mean & $3.14 \times 10^{-7}$ & 0.0159 & 0.2377 & 0.0181 & 0.0443 & 0.2568 & 0.0383 & 0.1012 & 0.2881 \\
\hline & Best & $2.28 \times 10^{-8}$ & 0.0158 & 0.2369 & $1.82 \times 10^{-5}$ & 0.0159 & 0.2387 & $6.19 \times 10^{-6}$ & 0.0159 & 0.2376 \\
\hline & Worst & $3.81 \times 10^{-6}$ & 0.0159 & 0.2385 & 0.0694 & 0.1637 & 0.3305 & 0.1616 & 0.2838 & 0.3957 \\
\hline & Std. & $7.39 \times 10^{-7}$ & $2.10 \times 10^{-5}$ & 0.0004 & 0.0219 & 0.0383 & 0.0241 & 0.0695 & 0.1264 & 0.0736 \\
\hline \multirow{4}{*}{$\xi$} & Mean & 2.0967 & 2.6818 & 5.0974 & 13.6510 & 18.8791 & 17.4444 & 4.2833 & 5.5063 & 5.9611 \\
\hline & Best & 0.1466 & 1.2389 & 0.2753 & 0.7383 & 2.1233 & 1.3675 & 0.3174 & 0.3636 & 1.5846 \\
\hline & Worst & 3.8125 & 4.9981 & 14.4016 & 49.3752 & 86.1865 & 55.5784 & 12.0010 & 12.5859 & 13.0184 \\
\hline & Std. & 1.3388 & 1.1167 & 3.1215 & 12.8726 & 18.4980 & 13.8440 & 4.2340 & 4.6146 & 4.2876 \\
\hline \multicolumn{2}{|c|}{ Algorithm } & \multicolumn{3}{|c|}{ ALO } & \multicolumn{3}{|c|}{ MVO } & \multicolumn{3}{|c|}{ SCA } \\
\hline \multicolumn{2}{|c|}{$\sigma_{v}^{2}$} & NN & 0.001 & 0.0025 & NN & 0.001 & 0.0025 & $\mathrm{NN}$ & 0.001 & 0.0025 \\
\hline \multirow{4}{*}{$J(\theta)$} & Mean & 0.0279 & 0.0739 & 0.2971 & 0.0129 & 0.0274 & 0.2814 & 0.0011 & 0.0194 & 0.2417 \\
\hline & Best & $5.07 \times 10^{-5}$ & 0.0159 & 0.2382 & $2.07 \times 10^{-6}$ & 0.0159 & 0.2376 & 0.0002 & 0.0168 & 0.2394 \\
\hline & Worst & 0.1619 & 0.2883 & 0.3952 & 0.1596 & 0.2783 & 0.3949 & 0.0023 & 0.0321 & 0.2495 \\
\hline & Std. & 0.0593 & 0.1086 & 0.0737 & 0.0439 & 0.0523 & 0.0704 & 0.0006 & 0.0033 & 0.0025 \\
\hline \multirow{4}{*}{$\xi$} & Mean & 4.9524 & 5.3427 & 7.1327 & 3.1334 & 3.4188 & 5.5603 & 2.6560 & 3.3897 & 3.6358 \\
\hline & Best & 0.4701 & 1.2324 & 0.4775 & 0.2459 & 1.2829 & 0.5581 & 0.4877 & 0.7339 & 1.0361 \\
\hline & Worst & 11.9252 & 11.5463 & 12.5844 & 11.5583 & 11.1893 & 12.8963 & 4.2392 & 7.9168 & 8.5802 \\
\hline & Std. & 3.4611 & 3.1596 & 3.4661 & 2.6600 & 1.9615 & 4.1303 & 1.1118 & 1.6111 & 2.0036 \\
\hline
\end{tabular}


Moreover, Table 3 tabulates the investigation of the statistical performance value of the objective function and the parameter estimation error with two noise variances, corresponding with no noise variances.

In this experiment, the mean, best, worst and Std. of the objective function and parameter estimation error are observed from the 25 trials at each level of noise variance. Specifically, the hMVOSCA based method produces better mean, best, worst and std. value of objective functions compared to the other methods for no noise and all level of noises. Moreover, the hMVOSCA based method also yields smaller mean and best values of parameter estimation error than the other methods for no noise and the noise variance $\boldsymbol{\sigma}_{v}^{2}=\mathbf{0 . 0 2 5}$. The statistical data illustrates that the hMVOSCA based method can effectively produce a relatively smaller parameter estimation error compared to the other methods even for different levels of noises.

The Wilcoxon's rank test result of the objective function of hMVOSCA with the other five algorithms for the TEC experiment is shown in Table 4. The tabular value shows that the proposed hMVOSCA based method has a significant difference compared with the PSO, GWO, ALO, MVO, and SCA based methods when there is no noise and for the other two noise variances. So, it is proven that the hMVOSCA based method has a significant difference in comparison with the other optimization methods.

Table 4. Wilcoxon's rank test of objective function of hMVOSCA and other five algorithms

\begin{tabular}{|c|c|c|c|c|c|c|}
\hline \multirow{2}{*}{ Noise } & \multirow{2}{*}{ Value } & \multicolumn{5}{|c|}{ hMVOSCA vs } \\
\cline { 3 - 7 } & & PSO & GWO & ALO & MVO & SCA \\
\hline \multirow{2}{*}{ No Noise } & $p$-value & $1.4157 \times 10^{-9}$ & $1.4157 \times 10^{-9}$ & $1.4157 \times 10^{-9}$ & $1.5967 \times 10^{-9}$ & $1.4157 \times 10^{-9}$ \\
\cline { 2 - 7 } & $h$-value & 1 & 1 & 1 & 1 & 1 \\
\hline \multirow{2}{*}{$\sigma_{v}^{2}=0.001$} & $p$-value & $1.4157 \times 10^{-9}$ & $1.5967 \times 10^{-9}$ & $1.4157 \times 10^{-9}$ & $1.8002 \times 10^{-9}$ & $1.4157 \times 10^{-9}$ \\
\cline { 2 - 7 } & $h$-value & 1 & 1 & 1 & 1 & 1 \\
\hline \multirow{2}{*}{$\sigma_{v}^{2}=0.0025$} & $p$-value & $1.4157 \times 10^{-9}$ & $2.8695 \times 10^{-8}$ & $2.0288 \times 10^{-9}$ & $1.1153 \times 10^{-6}$ & $1.4157 \times 10^{-9}$ \\
\cline { 2 - 7 } & $h$-value & 1 & 1 & 1 & 1 & 1 \\
\hline
\end{tabular}

\section{Conclusion}

A thermoelectric cooler plant identification using the continuous-time Hammerstein model based on the hybrid Multi-Verse Optimizer and Sine Cosine Algorithm (hMVOSCA) is presented in this paper. The results show that the proposed hMVOSCA based method has a good potential in identifying the behaviour of the TEC plant. In addition, from the perspective of quadratic output estimation error and parameter estimation error, the hMVOSCA based method is shown to be efficient in estimating both the linear and nonlinear subsystems of the Hammerstein model. Moreover, it is also shown that the hMVOSCA based method can generate a close estimated output with the output of the real thermoelectric cooler. This research can be applied as a potential direction of research to various types of nonlinear models, such as continuous-time Wiener model and Hammerstein-Wiener model. In contrast, the obtained results suggest that, compared to other optimization algorithms such as PSO, GWO, ALO, MVO and SCA, the proposed hMVOSCA produces better solutions. 
Acknowledgements: The authors would like to thank the University Malaysia Pahang for providing financial support under Internal Research Grant RDU182303.

\section{References}

1. S l a vov, T., A. Mit o v, J. Krale v. Advanced Embedded Control of Electrohydraulic Power Steering System. - Cybernetics and Information Technologies, Vol. 20, 2020, No 2, pp. 105-121.

2. K r a t m ü 11 e r, M. Real-Time Measurement System for High Temperature Drop Calorimeter. Cybernetics and Information Technologies, Vol. 10, 2010, No 1, pp. 64-71.

3. Stoyanov, B., V. Peichev, Y. Beyazov. Investigations on the Design of Discrete Pneumatic-to-Electrical Transducers of Low Pressure. - Cybernetics and Information Technologies, Vol. 8, 2008, No 1, pp. 65-72.

4. Li a n she ng, L. Research Progress on Alternative Refrigerants and Their Development Trend. J. of Refrigeration, Vol. 6, 2011.

5. L e e, M. Y., H. S. L e e, H. P. W o n. Characteristic Evaluation on the Cooling Performance of an Electrical Air Conditioning System Using R744 for a Fuel Cell Electric Vehicle. - Energies, Vol. 5, 2012, No 5, pp. 1371-1383.

6. Z h a n g, X. Research on Semiconductor Refrigeration System with Current Adaptive Temperature. - Advances in Engeneering Research, Vol. 148, 2017, pp. 53-56.

7. $\mathrm{H}$ u a n g, H., S. F u, P. Z h a n g, L. S u n. Design of a Small Temperature Control System Based on TEC. - In: Proc. of 9th Int. Symp. Comput. Intell. Des. Isc. 2016, Vol. 1, 2016, pp. 193-196.

8. H u, H. M., T. S. G e, Y. J. D a i, R. Z. W a n g. Experimental Study on Water-Cooled Thermoelectric Cooler for CPU under Severe Environment. - Int. J. of Refrigeration, Vol. 62, 2016, pp. 30-38.

9. A n d e r s e n, J. R. Thermoelectric Air Conditioner for Submarines. - Adv. Energy Convers, Vol. 2, 1962, pp. 241-248.

10. M a r 1 o w, R., R. J. B u i s t, J. L. N e 1 s o n. System Aspects of Thermoelectric Coolers for Hand Held Thermal Viewers, Garland, TX, US, Marlow Industries, Inc., 1982.

11. J u i, J. J., M. A. A h m a d. A Hybrid Metaheuristic Algorithm for Identification of ContinuousTime Hammerstein Systems. - Appl Math Model, Vol. 95, 2021, pp. 339-360.

12. J u i, J. J., M. H. S u id, M. R. Gh a z a li, M. A. A h m a d, M. Z. M. T u m a ri. Modified Sine Cosine Algorithm for Identification of Liquid Slosh Based on Continuous-Time Hammerstein Model. - J Phys Conf Ser, Vol. 1529, 2020, No 4, pp. 42-90.

13. L i, C. H., X. J. Z h u, G. Y. C a o, S. S u i, M. R. H u. Identification of the Hammerstein Model of a PEMFC Stack Based on Least Squares Support Vector Machines. - J. Power Sources, Vol. 175, 2008, No 1, pp. 303-316.

14. Zhang, Q., Q. Wang, G. Li. Nonlinear Modeling and Predictive Functional Control of Hammerstein System with Application to the Turntable Servo System. - Mech. Syst. Signal Process, Vol. 72-73, 2016, pp. 383-394.

15. S a l e e m, A., M. M e s b a h, S. A l-R a t o u t. Nonlinear Hammerstein Model Identification of Amplified Piezoelectric Actuators (APAs): Experimental Considerations. - In: Proc. of 4th International Conference on Control, Decision and Information Technologies (CoDIT'17), 2017, pp. 633-638.

16. Z h a n g, H. T., B. H u, L. L i, Z. C h e n, D. W u, B. X u et al. Distributed Hammerstein Modeling for Cross-Coupling Effect of Multiaxis Piezoelectric Micropositioning Stages. - IEEE/ASME Trans Mechatronics, Vol. 23, 2018, No 6, pp. 2794-2804.

17. A i, Q., Y. P e n g, J. Z u o, W. M e n g, Q. Liu. Hammerstein Model for Hysteresis Characteristics of Pneumatic Muscle Actuators. - Int. J. Intell. Robot. Appl., Vol. 3, 2019, No 1, pp. 33-44.

18. Hou, J., F. Che n, P. Li, Z. Zhu. Fixed Point Iteration-Based Subspace Identification of Hammerstein State-Space Models. - IET Control Theory Appl., Vol. 13, 2019, No 8, pp. 1173-1181.

19. H o u, J., T. L i u, Q. G. W a n g. Subspace Identification of Hammerstein-Type Nonlinear Systems Subject to Unknown Periodic Disturbance. - Int. J. Control, 2019, pp. 1-11. 
20. D i n g, F., H. Ch e n, L. X u, J. D a i, Q. L i, T. H a y a t. A Hierarchical Least Squares Identification Algorithm for Hammerstein Nonlinear Systems Using the Key Term Separation. - J. of the Franklin Inst., Vol. 355, 2018, No 8, pp. 3737-3752.

21. W a n g, J., A. S a n o, T. Ch e n, B. H u a n g. A Blind Approach to Identification of Hammerstein Systems. - In: Lect. Notes in Control and Inf. Sci., Vol. 404. 2010, pp. 293-312.

22. G o t $\mathrm{m}$ a r e, A., R. P a t i d a r, N. V. G e o r g e. Nonlinear System Identification Using a Cuckoo Search Optimized Adaptive Hammerstein Model. - Expert. Syst. Appl., Vol. 42, 2015, No 5, pp. 2538-2546.

23. A 1-D u w a i s h, H. N. Identification of Hammerstein Models with Known Nonlinearity Structure Using Particle Swarm Optimization. - Arab. J. of Sci. Eng., Vol. 36, 2011, No 7, pp. 1269-1276.

24. Cuevas, E., P. Día z, O. Avalos, D. Zaldívar, M. Pérez-Cisneros, DE C P et al. Nonlinear System Identification Based on ANFIS-Hammerstein Model Using Gravitational Search Algorithm. - Appl. Intell., Vol. 48, 2018, No 1, pp. 182-203.

25. J u i, J. J., M. H. S u i d, Z. M u s a, M. A. A h m a d. Identification of Liquid Slosh Behavior Using Continuous-Time Hammerstein Model Based Sine Cosine Algorithm. - In: Proc. of 11th National Technical Seminar on Unmanned System Technology (NUSYS'19), pp. 345-356.

26. M ir ja 1 i li, S., S. M. M ir jali li, A. H a t a m 1 o u. Multi-Verse Optimizer: A Nature-Inspired Algorithm for Global Optimization. - Neural Comput. Appl., Vol. 27, 2016, No 2, pp. 495-513.

27. Ju i, J. J., M. A. Ahmad, M. I. M. Rashid. Modified Multi-Verse Optimizer for Solving Numerical Optimization Problems. - In: Proc. of IEEE Int. Conf. Autom. Control Intell. Syst. (I2CACIS'20), 2020, pp. 81-86.

28. A 1 i, E. E., M. A. E 1-H a m e e d, A. A. E 1-F e r g a n y, M. M. E 1-A r in i. Parameter Extraction of Photovoltaic Generating Units Using Multi-Verse Optimizer. - Sustain Energy Technol. Assessments, Vol. 17, 2016, pp. 68-76.

29. J a n g i r, P., S. A. Parm ar, I. N. Trivedi, R. H. B he s d a di y a. A Novel Hybrid Particle Swarm Optimizer with Multi Verse Optimizer for Global Numerical Optimization and Optimal Reactive Power Dispatch Problem. - Eng. Sci. Technol. an Int. J., Vol. 20, 2017, No 2, pp. 570-586.

30. G u h a, D., P. K. R o y, S. B a n e r j e e. Multi-Verse Optimisation: A Novel Method for Solution of Load Frequency Control Problem in Power System. - IET Gener. Transm. Distrib., Vol. 11, 2017, No 14, pp. 3601-3611.

31. F a th y, A., H. R e z k. Multi-Verse Optimizer for Identifying the Optimal Parameters of PEMFC Model. - Energy, Vol. 143, 2018, pp. 634-644.

32. W a n g, X., D. L u o, X. Z h a o, Z. S u n. Estimates of Energy Consumption in China Using a SelfAdaptive Multi-Verse Optimizer-Based Support Vector Machine with Rolling CrossValidation. - Energy, Vol. 152, 2018, pp. 539-548.

33. M i r j a 1 i li, S. SCA: A Sine Cosine Algorithm for Solving Optimization Problems. - KnowledgeBased Syst., 2016, pp. 120-133.

34. Abd Elaziz, M. E., A. A. E wees, D. Oliva, P. Du an, S. Xi on g. A Hybrid Method of Sine Cosine Algorithm and Differential Evolution for Feature Selection. - In: Lect. Notes in Comput. Sci. Vol. 10638. 2017, pp. 145-155.

35. A l-Q a ne s s, M. A. A., M. A. E 1 a z i z, A. A. E w e e s. Oil Consumption Forecasting Using Optimized Adaptive Neuro-Fuzzy Inference System Based on Sine Cosine Algorithm. - IEEE Access, Vol. 6, 2018, pp. 68394-68402.

36. Oliva, D., S. Hi nojos a, M. A. Elaziz, N. Ortega-Sánchez. Context Based Image Segmentation Using Antlion Optimization and Sine Cosine Algorithm. - Multimed. Tools Appl., Vol. 77, 2018, No 19, pp. 25761-25797.

37. S a y e d, G. I., A. D a r w i s h, A. E. H a s s a n i e n. Quantum Multiverse Optimization Algorithm for Optimization Problems. - Neural Comput. Appl., Vol. 31, 2019, No 7, pp. 2763-2780.

38. Huang, B. J., C. L. Duang. System Dynamic Model and Temperature Control of a Thermoelectric Cooler. - Int. J. of Refrigeration, Vol. 23, 2000, No 3, pp. 197-207.

Received: 27.01.2021; Second Version: 01.07.2021; Third Version: 26.07.2021; Accepted: 10.08.2021 